\title{
HYPERBOLIC POLYNOMIALS AND SPECTRAL ORDER
}

\author{
JULIUS BORCEA AND BORIS SHAPIRO
}

\begin{abstract}
The spectral order on $\mathbb{R}$ induces a partial ordering on the manifold $\mathcal{H}_{n}$ of monic hyperbolic polynomials of degree $n$. We show that the semigroup $\tilde{\mathcal{S}}$ generated by differential operators of the form $\left(1-\lambda \frac{d}{d x}\right) e^{\lambda \frac{d}{d x}}, \lambda \in \mathbb{R}$, acts on the poset $\mathcal{H}_{n}$ in an order-preserving fashion. We also show that polynomials in $\mathcal{H}_{n}$ are global minima of their respective $\tilde{\mathcal{S}}$-orbits and we conjecture that a similar result holds even for complex polynomials. Finally, we show that only those pencils of polynomials in $\mathcal{H}_{n}$ which are of logarithmic derivative type satisfy a certain local minimum property for the spectral order.
\end{abstract}

\section{Introduction And Main Results}

Given a complex polynomial $P$ of degree $n$ we define $Z(P)$ to be the unordered $n$-tuple consisting of the zeros of $P$, where each zero occurs as many times as its multiplicity. We denote by $\Re Z(P)$ the (unordered) $n$-tuple consisting of the real parts of the points in $Z(P)$. The polynomial $P$ is said to be hyperbolic if all its zeros are real. Note that in this case $\Re Z(P)=Z(P)$. A hyperbolic polynomial whose zeros are simple is called strictly hyperbolic.

The main purpose of this paper is to study the behaviour of the $n$-tuple $Z(P)$ under the action of certain semigroups of differential operators. For this we shall use the following fundamental result from the theory of stochastic majorizations:

Theorem 1. Let $X=\left(x_{1}, x_{2}, \ldots, x_{n}\right)^{t}$ and $Y=\left(y_{1}, y_{2}, \ldots, y_{n}\right)^{t}$ be two unordered $n$-tuples of vectors in $\mathbb{R}^{k}$. The following conditions are equivalent:

(1) For any convex function $f: \mathbb{R}^{k} \rightarrow \mathbb{R}$ one has $\sum_{i=1}^{n} f\left(x_{i}\right) \leq \sum_{i=1}^{n} f\left(y_{i}\right)$.

(2) There exists a doubly stochastic $n \times n$ matrix $A$ such that $\tilde{X}=A \tilde{Y}$, where $\tilde{X}$ and $\tilde{Y}$ are $n \times k$ matrices obtained by some (and then any) ordering of the vectors in $X$ and $Y$.

If the conditions of Theorem 1 1 are satisfied then we say that $X$ is majorized by $Y$ or $X$ is less than $Y$ in the spectral order, and write $X \prec Y$. Theorem 1 is due to Schur and to Hardy, Littlewood and Pólya in the one-dimensional case ( $\underline{\mathrm{HLP}})$, and to Sherman in the multivariate case $(\underline{\mathrm{S}} \mid)$. These cases are also known as classical and multivariate majorization, respectively. One can easily check that $X \prec Y$ implies that $\sum_{i=1}^{n} x_{i}=\sum_{i=1}^{n} y_{i}$.

Let $\mathcal{H}_{n}$ denote the manifold of monic hyperbolic polynomials of degree $n$. We may view $\left(\mathcal{H}_{n}, \preccurlyeq\right)$ as a partially ordered set, where the ordering relation $\preccurlyeq$ is induced by the spectral order on $n$-tuples of real numbers (cf. Theorem 11). Thus, if $P, Q \in$ $\mathcal{H}_{n}$ then $P \preccurlyeq Q$ if and only if $Z(P) \prec Z(Q)$. Note that although the spectral order is only a preordering on $n$-tuples of points in $\mathbb{R}$, Birkhoff's theorem (MO Theorem 2.A.2]) implies that it actually induces a partial ordering on $\mathcal{H}_{n}$.

Define the following semigroups of differential operators:

$$
\mathcal{S}=\left\langle 1-\lambda \frac{d}{d x} \mid \lambda \in \mathbb{R}\right\rangle, \quad \tilde{\mathcal{S}}=\left\langle\left(1-\lambda \frac{d}{d x}\right) e^{\lambda \frac{d}{d x}} \mid \lambda \in \mathbb{R}\right\rangle .
$$

2000 Mathematics Subject Classification. Primary 30C15; Secondary 60E15. 
Note that $\tilde{\mathcal{S}}$ is the (largest) subsemigroup of $\mathcal{S} \times\left\langle e^{\mu \frac{d}{d x}} \mid \mu \in \mathbb{R}\right\rangle$ which consists of operators that preserve the averages of the zeros of polynomials in $\mathcal{H}_{n}$. The operator $\left(1-\lambda \frac{d}{d x}\right) e^{\lambda \frac{d}{d x}}, \lambda \in \mathbb{R}$, will be denoted by $D_{\lambda}$ throughout this paper. It follows from the well-known Hermite-Poulain theorem (see [0] that the semigroups $\mathcal{S}$ and $\tilde{\mathcal{S}}$ act on $\mathcal{H}_{n}$. Our first main result asserts that these semigroups act in fact on $\mathcal{H}_{n}$ in an order-preserving fashion:

Theorem 2. Let $P, Q \in \mathcal{H}_{n}$ be such that $P \preccurlyeq Q$. Then $P+\lambda P^{\prime} \preccurlyeq Q+\lambda Q^{\prime}$ for any $\lambda \in \mathbb{R}$.

We point out an interesting consequence of Theorem 2

Corollary 1. If $P, Q \in \mathcal{H}_{n}$ are such that $P \preccurlyeq Q$ then $n^{-1} P^{\prime} \preccurlyeq n^{-1} Q^{\prime}$.

The next theorem shows that any polynomial in $\mathcal{H}_{n}$ is the global minimum of its orbit under the action of the semigroup $\tilde{\mathcal{S}}$.

Theorem 3. If $P \in \mathcal{H}_{n}$ then $P \preccurlyeq D_{\lambda} P$ for any $\lambda \in \mathbb{R}$.

A well-known theorem of Obreschkoff (see [0]) states that if $P$ and $Q$ are real polynomials then the linear pencil of polynomials $P+\lambda Q, \lambda \in \mathbb{R}$, consists of hyperbolic polynomials if and only if $P$ and $Q$ are hyperbolic and those of their zeros which are not common separate each other. The following converse to Theorem 3 shows that real lines in $\mathcal{H}_{n}$ of the form $P+\lambda P^{\prime}$ are characterized by a local minimum property with respect to the partial ordering $\preccurlyeq$ on $\mathcal{H}_{n}$.

Theorem 4. Let $P \in \mathcal{H}_{n}, \lambda \in \mathbb{R}$, and let $Q$ be a complex polynomial of degree at most $n-1$. Set $R_{\lambda}(x)=P(x+\lambda)-\lambda Q(x+\lambda)$. If $R_{\lambda} \in \mathcal{H}_{n}$ and $R_{0} \preccurlyeq R_{\lambda}$ for all small $\lambda \in \mathbb{R}$ then $Q=P^{\prime}$.

We also obtain a generalization of Theorem 3 which shows that real lines in $\mathcal{H}_{n}$ of the form $P+\lambda P^{\prime}$ satisfy in fact a global monotony property:

Theorem 5. If $P \in \mathcal{H}_{n}$ and $\lambda_{1}, \lambda_{2} \in \mathbb{R}$ are such that $\lambda_{1} \lambda_{2} \geq 0$ and $\left|\lambda_{1}\right| \leq\left|\lambda_{2}\right|$ then $D_{\lambda_{1}} P \preccurlyeq D_{\lambda_{2}} P$.

Finally, we show that real lines in $\mathcal{H}_{n}$ of the form $P+\lambda P^{\prime}$ satisfy an inequality à la Gårding (cf. G]):

Theorem 6. If $P \in \mathcal{H}_{n}$ then $\mathbb{R} \ni \lambda \mapsto \max Z\left(D_{\lambda} P\right)$ is a convex function with a global minimum at $\lambda=0$ while $\mathbb{R} \ni \lambda \mapsto \min Z\left(D_{\lambda} P\right)$ is a concave function with a global maximum at $\lambda=0$.

Remark 1. It follows from Theorem 6 that the so-called spread function $\mathbb{R} \ni \lambda \mapsto$ $\max Z\left(D_{\lambda} P\right)-\min Z\left(D_{\lambda} P\right)$ is a convex function with a global minimum at $\lambda=0$.

The structure of the paper is as follows: in $\S 1$ we sketch the proofs of our main results and in $\S 2$ we present further questions and conjectures. The complete proofs will appear elsewhere.

\section{Outline of the PROOFS}

One of the key ingredients in the proofs of Theorems 26 is the following criterion for classical majorization due to Hardy, Littlewood and Pólya (cf. HLP]). We should mention that there are no known analogues of this criterion for multivariate majorization.

Theorem 7. Let $X=\left(x_{1} \leq x_{2} \leq \ldots \leq x_{n}\right) \subset \mathbb{R}$ and $Y=\left(y_{1} \leq y_{2} \leq \ldots \leq y_{n}\right)$ be two n-tuples of real numbers. Then $X \prec Y$ if and only if the $x_{i}$ 's and the $y_{i}$ 's satisfy the following conditions: 
(1) $\sum_{i=1}^{n} x_{i}=\sum_{i=1}^{n} y_{i}$;

(2) $\sum_{i=0}^{k} x_{n-i} \leq \sum_{i=0}^{k} y_{n-i}$ for $0 \leq k \leq n-2$.

The proof of Theorem 2 is based on several auxiliary results. Let us first make the following definition:

Definition 1. Let $P(x)=\prod_{i=1}^{n}\left(x-x_{i}\right) \in \mathcal{H}_{n}, n \geq 2$, and $1 \leq k<l \leq n$. Assume that $x_{i} \leq x_{i+1}, 1 \leq i \leq n-1$, and that $x_{k} \neq x_{l}$. Let further $\left.\left.t \in\right] 0, \frac{x_{l}-x_{k}}{2}\right]$ and define $Q \in \mathcal{H}_{n}$ to be the polynomial with zeros $y_{i}, 1 \leq i \leq n$, where $y_{k}=x_{k}+t$, $y_{l}=x_{l}-t$, and $y_{i}=x_{i}, i \neq k, l$. The polynomial $Q$ is called the contraction of $P$ of type $(k, l)$ and coefficient $t$. The contraction is called simple if $l=k+1$ and it is called non-degenerate if $t \neq \frac{x_{l}-x_{k}}{2}$.

Proposition 1. Let $P, Q \in \mathcal{H}_{n}$ be two distinct strictly hyperbolic polynomials such that $P \preccurlyeq Q$. Then there exists a finite sequence $P_{1}, \ldots, P_{m} \in \mathcal{H}_{n}$ such that $P_{1}=Q$, $P_{m}=P$, and $P_{i+1}$ is a simple non-degenerate contraction of $P_{i}, 1 \leq i \leq m-1$.

Remark 2. Proposition [1 is true even for polynomials with multiple zeros if the non-degeneracy condition is omitted.

Proposition 2. Theorem 2 is true if $P$ and $Q$ are strictly hyperbolic polynomials and $P$ is a simple (non-degenerate) contraction of $Q$.

From Propositions 1 and 2 we deduce that Theorem 2 is true in the generic case when $P$ and $Q$ have simple zeros. If this is not the case then we let $x_{i}$, $1 \leq i \leq n$, and $y_{i}, 1 \leq i \leq n$, denote the zeros of $P$ and $Q$, respectively, and we choose an arbitrary positive number $\varepsilon$. Let $P_{\varepsilon}$ and $Q_{\varepsilon}$ be the polynomials with zeros $x_{i}-(n-i) \varepsilon, 1 \leq i \leq n-1, x_{n}+\frac{n(n-1)}{2} \varepsilon$, and $y_{i}-(n-i) \varepsilon, 1 \leq i \leq n-1$, $y_{n}+\frac{n(n-1)}{2} \varepsilon$, respectively. Note that $P_{\varepsilon}$ and $Q_{\varepsilon}$ are strictly hyperbolic and that $P_{\varepsilon} \preccurlyeq Q_{\varepsilon}$. The above arguments imply that $P_{\varepsilon}+\lambda P_{\varepsilon}^{\prime} \preccurlyeq Q_{\varepsilon}+\lambda Q_{\varepsilon}^{\prime}$ for any $\lambda \in \mathbb{R}$. Theorem 2 now follows by letting $\varepsilon \rightarrow 0$.

Let $P(x)=\prod_{i=1}^{n}\left(x-x_{i}\right) \in \mathcal{H}_{n}, n \geq 2$, and $\lambda \in \mathbb{R}$. Assume that $x_{i}<x_{i+1}$, $1 \leq i \leq n-1$, and let $x_{i}(\lambda), 1 \leq i \leq n$, denote the zeros of $D_{\lambda} P$. If these are labeled so that $x_{i}(0)=x_{i}, 1 \leq i \leq n$, then one can show that $x_{i}(\lambda)<x_{i+1}(\lambda)$ and that by varying $x_{n}$ and keeping $\lambda$ fixed each $x_{i}(\lambda), 1 \leq i \leq n-1$, is an increasing function of $x_{n}$. This makes it possible to prove Theorem 3 by induction on $n$ in the generic case. If $P \in \mathcal{H}_{n}$ has multiple zeros we notice that $\left(1-\varepsilon \frac{d}{d x}\right)^{n-1} P$ has simple zeros for any $\varepsilon \neq 0$. Since Theorem 3 is true for the latter polynomials, we get that Theorem 3 holds in the general case by letting $\varepsilon \rightarrow 0$.

To prove Theorem 4 we first use Theorem 7 in order to show that if $P$ is strictly hyperbolic and $Q$ satisfies the assumptions of Theorem 4 then $Q\left(x_{i}\right)=P^{\prime}\left(x_{i}\right)$, where $x_{i}, 1 \leq i \leq n$, are the zeros of $P$. Using again the fact that $\left(1-\varepsilon \frac{d}{d x}\right)^{n-1} P$ has simple zeros if $P \in \mathcal{H}_{n}$ and $\varepsilon \neq 0$ and also that the operator $\left(1-\varepsilon \frac{d}{d x}\right)^{n-1}$ preserves the ordering on $\mathcal{H}_{n}$ (cf. Theorem 2) we deduce that Theorem 4 holds for any $P \in \mathcal{H}_{n}$.

Let now $P(x)=\prod_{i=1}^{n}\left(x-x_{i}\right) \in \mathcal{H}_{n}, n \geq 2, \lambda \in \mathbb{R}$, and assume that $x_{i}<x_{i+1}$, $1 \leq i \leq n-1$. Denote the zeros of $D_{\lambda} P$ by $x_{i}(\lambda), 1 \leq i \leq n$, those of $P^{\prime}$ by $w_{j}$, $1 \leq j \leq n-1$, and those of $D_{\lambda} P^{\prime}$ by $w_{j}(\lambda), 1 \leq j \leq n-1$. If these are labeled so that $x_{i}(0)=x_{i}, 1 \leq i \leq n$, and $w_{j}(0)=w_{j}, 1 \leq j \leq n-1$, then one can show that $x_{i}(\lambda)<w_{i}(\lambda)<x_{i+1}(\lambda), 1 \leq i \leq n-1$, and that for any $\lambda>0$ and $1 \leq m \leq n-1$ one has

$$
\sum_{i=1}^{m} x_{i}^{\prime}(\lambda)=\lambda \sum_{j=1}^{n-1} \frac{P^{\prime \prime}\left(w_{j}(\lambda)+\lambda\right)}{D_{\lambda} P^{\prime \prime}\left(w_{j}(\lambda)\right)}\left(\sum_{i=1}^{m} \frac{1}{x_{i}(\lambda)-w_{j}(\lambda)}\right)<0 .
$$


Thus $\lambda \mapsto \sum_{i=1}^{m} x_{i}(\lambda), 1 \leq m \leq n-1$, are decreasing functions on ]0, $\infty$ [, which combined with Theorem 7 proves Theorem [5] for strictly hyperbolic polynomials (the case $\lambda<0$ is similar). The same density arguments as those used for Theorem 4 show that Theorem 5 is true for all $P \in \mathcal{H}_{n}$.

The second part of the statement in Theorem 6 follows from the first part by noticing that $P(x) \in \mathcal{H}_{n}$ if and only if $(-1)^{n} P(-x) \in \mathcal{H}_{n}$. Keeping the same notations as above, one can show that if $P \in \mathcal{H}_{n}$ is strictly hyperbolic then

$$
x_{n}^{\prime \prime}(\lambda)=2\left(x_{n}^{\prime}(\lambda)+1\right)^{2} \sum_{i=1}^{n-1} \frac{w_{i}-x_{i}(\lambda)-\lambda}{\left(x_{n}(\lambda)+\lambda-w_{i}\right)\left(x_{n}(\lambda)-x_{i}(\lambda)\right)}>0
$$

for any $\lambda \in \mathbb{R}$, which proves Theorem [6] in the generic case. If $P$ has multiple zeros then one can use strictly hyperbolic polynomials of the form $\left(1-\frac{1}{s} \frac{d}{d x}\right)^{n-1} P$, $s \in \mathbb{Z}_{+}$, in order to approximate the function $\lambda \mapsto \max Z\left(D_{\lambda} P\right)$ uniformly on compact intervals by convex $C^{2}$-functions. This proves Theorem 6 .

\section{RemARKS AND OPEN QUESTIONS}

The manifold $\mathcal{C}_{n}$ of monic complex polynomials of degree $n$ is a natural context for discussing possible extensions of the above results to the complex case. By analogy with the hyperbolic case we may view $\left(\mathcal{C}_{n}, \preccurlyeq\right)$ as a partially ordered set, where the ordering relation $\preccurlyeq$ is now induced by the spectral order on $n$-tuples of vectors in $\mathbb{R}^{2}$ (cf. Theorem 11). This means that the zero sets of polynomials in $\mathcal{C}_{n}$ are viewed as subsets of $\mathbb{R}^{2}$ and that if $P, Q \in \mathcal{C}_{n}$ then $P \preccurlyeq Q$ if and only if $Z(P) \prec Z(Q)$.

The following example shows that if the partial ordering $\preccurlyeq$ on $\mathcal{C}_{n}$ is defined as above then one cannot expect a complex analogue of Theorem [3]

Proposition 3. Let $P(z)=z^{n}-1$ and $\lambda \in \mathbb{C}$. If $n \geq 3$ and $|\lambda|$ is small enough then $D_{\lambda} P$ and $P$ are incomparable with respect to the partial ordering $\preccurlyeq$ on $\mathcal{C}_{n}$.

We also note that the results of the previous section (for the hyperbolic case) are valid only for real values of the parameter $\lambda$ :

Proposition 4. Let $P \in \mathcal{H}_{n}$ be a strictly hyperbolic polynomial and let $\lambda \in \mathbb{C} \backslash \mathbb{R}$. If $n \geq 2$ and $|\lambda|$ is sufficiently small then $D_{\lambda} P$ and $P$ are incomparable with respect to the partial ordering $\preccurlyeq$ on $\mathcal{C}_{n}$.

These examples suggest that complex generalizations of Theorem 3 - if any should involve only classical majorization and real values of the parameter $\lambda$. Based on extensive numeric calculations, we make the following

Conjecture 1. If $P \in \mathcal{C}_{n}$ then $\Re Z(P) \preccurlyeq \Re Z\left(D_{\lambda} P\right)$ for any $\lambda \in \mathbb{R}$.

We end with a few questions related to the semigroups $\mathcal{S}$ and $\tilde{\mathcal{S}}$. Let $\mathcal{R}_{n}$ denote the set of all monic real polynomials of degree $n$. There is reason to believe that any two $\mathcal{S}$-orbits in $\mathcal{R}_{n}$ have a non-empty intersection:

Conjecture 2. If $P_{1}, P_{2} \in \mathcal{R}_{n}$ then there exist differential operators $\Lambda_{1}, \Lambda_{2} \in \mathcal{S}$ such that $\Lambda_{1} P_{1}=\Lambda_{2} P_{2}$.

If true, Conjecture 2 would imply in particular that $\mathcal{S} P \cap \mathcal{H}_{n} \neq \emptyset$ for any $P \in \mathcal{R}_{n}$, which would answer in the affirmative a question of I. Krasikov.

Let finally $P \in \mathcal{H}_{n}$ and set $P_{\preccurlyeq}=\left\{Q \in \mathcal{H}_{n} \mid P \preccurlyeq Q\right\}$. One can easily check that if $P$ is strictly hyperbolic and $n \geq 3$ then $\tilde{\mathcal{S}} P \subsetneq P_{\preccurlyeq}$. It would be interesting to know whether there exists a (semi)group $\mathcal{D}$ of differential operators (not necessarily with constant coefficients) such that $\mathcal{D} \supsetneq \tilde{\mathcal{S}}$ and $P_{\preccurlyeq}=\mathcal{D} P$ for any $P \in \mathcal{H}_{n}$. This would give a completely new way of describing classical majorization. 
Acknowledgements. The results announced in this paper and their proofs were all obtained by the first author (JB). The second author (BS) only made some computer tests in connection with Remark 1 and Theorem [3. Both authors are grateful to Harold Shapiro and Ilia Krasikov for stimulating discussions.

\section{REFERENCES}

[G] Gårding, L., An inequality for hyperbolic polynomials, J. Math. Mech. 8 (1959), 957-965.

[HLP] Hardy, G. H., Littlewood, J. E., and Pólya, G., Inequalities, Cambridge University Press, 2nd ed., 1988.

[MO] Marshall, A. W., Olkin, I., Inequalities: Theory of Majorization and Its Applications, Mathematics in Science and Engineering vol. 143, Academic Press, New York, 1979.

[O] Obreschkoff, N., Verteilung und Berechnung der Nullstellen reeller Polynome, VEB Deutscher Verlag der Wissenschaften, 1963.

[S] Sherman, S., On a theorem of Hardy, Littlewood, Pólya, and Blackwell, Proc. Nat. Acad. Sci. USA 37 (1951), 826-831.

Department of Mathematics, Stockholm University, SE-106 91 Stockholm, Sweden

E-mail address: julius@math.su.se

Department of Mathematics, Stockholm University, SE-106 91 Stockholm, Sweden

E-mail address: shapiro@math.su.se 